\title{
The Cost-Based Lean Approach to the Information Logistics Business System Modelling
}

\author{
Robert Bucki and Petr Suchánek \\ School of Business Administration in Karvina, Silesian University in Opava, Czech Republic
}

\begin{abstract}
The paper highlights the problems of modelling the complex logistics manufacturing system which is to meet the needs of the contemporary lean approach to manufacturing. It is assumed that the system consists of identically arranged and equipped subsystems, however, each of them is characterised by different operating and fixed costs. The software engineering method used to create the simulator of the discussed system is emphasised to show how the necessary assumptions turn the real manufacturing system into the mathematical model of the logistic-business system, and subsequently into the simulator. Manufacturing strategies as well as heuristic algorithms are introduced in order to control making the order. The case study presents the method of searching for the most adequate manufacturing subsystem for making the order on the basis of cost calculation. The key points responsible for excessive costs are detected. A thorough analysis based on modified operating as well as fixed costs is carried out.
\end{abstract}

ACM CCS (2012) Classification: Computing methodologies $\rightarrow$ Artificial intelligence $\rightarrow$ Distributed artificial intelligence $\rightarrow$ Multi-agent systems

Computing methodologies $\rightarrow$ Modeling and simulation $\rightarrow$ Model development and analysis $\rightarrow$ Modeling methodologies

Keywords: logistics system, mathematical modelling, simulation, business process, manufacturing strategies, optimisation

\section{Introduction}

Contemporary business environment is evolving rapidly, requiring more and more regular regulatory intervention from managers, which should be effective and increasingly less error-prone. New means of support are sought and developed to provide the information that is required before the correct decision can be made. Modern - and now standard - tools to support decision-making, management, and optimisation of different types of systems include software tools which enable simulation processes to be carried out [1]. Simulations are based on mathematical models whose correct construction and final definition along with input data are the primary prerequisite for correct and usable results of individual simulations to be obtained. Simulations can be used for existing systems or systems which do not exist yet, such as a design or implementation plan. Simulations of existing systems are being implemented in order to detect their weaknesses and to make their activities more efficient. This can usually be achieved by adjusting the overall architecture or partial architectures (physical, logical, data, functional, etc.) or its sub-processes [2], [3]. The adequate simulation model is made up of a mathematical model which contains mathematical expression of structure (formalisation), parameters of system elements, limiting conditions and decision algorithms (means, evaluation methods, etc.). The mathematical and subsequent simulation models are based on the system being simulated. Examples of good practice and theoretical bases for creating descriptive, mathematical and simulation models of production, logistics, business or generally socio-economic or other systems include, for example, Petri-based approaches [4], [5], [6], the multi-agent approach [7], [8], [9], [10], the hybrid approach [11], [12], the 
distributed approach [13], stochastic methods [14], [15], the heuristic approach [16], [17], [18], [19], neural networks [20], [21], [22] and others. Each of these approaches has different advantages, disadvantages and limitations for a particular situation. The heuristic approach has been chosen for the purpose of this paper as the most appropriate, however, it is not always possible to find the best solution. Nevertheless, the satisfactory solution is often achievable in a short time and, more importantly, it is relatively easy to apply. The second method that would be useful to implement is a multi-agent approach that would allow wider possibilities in determining the properties of individual objects and their variants in the system defined for the purpose of the article. As mentioned, the mathematical-statistical methods are the means by which structures, architecture resp. models of each system are defined and evaluated [23], [24]. One of the decisive simulation factors is good knowledge of the system and the set of monitored parameters. Time and price are the most frequently used parameters for simulation of production logistics systems and general business processes. These two parameters are crucial for the evaluation and re-engineering of processes from an economic point of view and simulations work relatively well in this case. These are parameters that, besides others, are directly linked to the concept of the so-called lean company (or lean manufacturing, lean logistics, etc.) and Kanban [25], [26], [27], [28], [29]). It should be noted that this paper deals with efficiency issues by automatically assuming that discounting or shortening the time of production, distribution or other business processes never leads to a decrease in quality. Special software or sophisticated mathematical calculating tools are used to carry out the simulation process. When creating a simulation module, it is necessary to implement standard software development methodologies e.g. very often agile methodologies are used [30].

Increasing the effectiveness of classical mathematical models and simulators based on heuristic approaches and algorithms can be achieved by implementing the theory and practice of multiagent systems. The reason for this is that in a manufacturing environment which is architecturally understood as a serial production system, production lines are usually a set of in- dividual elements (i.e. robots, cobots, people) that implement a specific set of operations in a precisely defined sequence. Once they have been executed, the intermediate product is passed to another element. The final manufacturing workstation in the production line is the final product. Individual elements can perform all required manufacturing operations if all necessary conditions for their implementation are met (e.g. element functionality, availability of all resources, etc.). If the required condition is not met at any given time, a problem arises because the element is unable to complete the production operation and send the intermediate product to the subsequent workstation. The basic research questions in this context are how to minimize these conditions, how to solve them as quickly as possible and, ideally, predict them. Here it is very advantageous to extend the local intelligence of the individual elements, which can be made precisely by means of methodical procedures typical of multiagent systems. In this case, there is an ordered set of production elements built as a multiagent system where each agent is treated as an autonomous unit able to react with other adjacent agents (e.g. intermediate delivery, current status) as well as proactive ones (e.g. durability of production tools). Increasing the local intelligence of individual elements significantly increases the intelligence of the entire complex production system. Therefore, the use of multiagent systems is a significant trend in mathematical models, simulators and, of course, real practice which opens new opportunities for optimizing and increasing intelligence and production efficiency in the future.

Sample processes described in this paper by the mathematical modelling method have their roots in the automotive industry which is a wide range of companies and organisations involved in the numerous activities including, among others, design, development, manufacturing, marketing, and selling of motor vehicles. The problem presented in this paper takes into account the knowledge emerging from the real manufacturing environment. However, for the simplification needs, it is necessary to bear in mind that the paper focuses on illustrating the modelling method. Processes which are subject to a thorough analysis take place in the sample real manufacturing environment of a supplier 
company for the automotive industry which has its branches in various locations across the globe. It is meant to guarantee continuous supplies to its final customers without any standstills which would disturb their continuous manufacturing process. The main goal of the supplier company is to minimise operating and fixed costs and secure the manufacturing processes. Often, the company manufactures identical products in distant locations, however, the headquarters' managers tend to arrange manufacturing processes in their sub-companies in the same way. Thus it is possible to compare the sub-companies and locate customers' orders in the most profitable sub-company. This kind of approach results from different labour force costs, various costs of charge materials and different storage costs, for example. Nevertheless, it is important to take into account manufacturing security which is not to be neglected in the changing geopolitical environment. To illustrate the complexity of the problem, it is necessary to carry out the whole modelling process beginning with the real system analysis. A similar approach can be found in [31].

The main goal of this paper is to analyse the problems of modelling the complex logistics manufacturing system which is to meet the needs of the contemporary lean approach to production matters. It requires introducing the mathematical model of the logistics manufacturing system to solve sophisticated manufacturing tasks. The subsequent goal of the paper is to present the method of choosing the subsystem which minimises the total manufacturing costs. This course of action can be carried out in an illustrative way by means of the thorough analysis during the case study procedure of the sample multi-plant system functioning. The software engineering method used to create the simulator of the discussed system is emphasised to show how the necessary assumptions turn the real manufacturing system into the mathematical model of the logistic-business system. First of all, assumptions based on the thorough analysis of the real system enable us to build the mathematical model of the logistics manufacturing system. In order to model the system, mathematical foundations must be put forward for all necessary interdependencies in the system consisting of the defined number of identically arranged and equipped subsystems, however, each of them is characterised by different operating and fixed costs. Subsequently, manufacturing strategies, along with heuristic control algorithms, are introduced with the aim of controlling the order making process. The iteration method for creating the adequate simulation tool is presented in detail. Moreover, the dedicated simulator of the logistics manufacturing system is described. Finally, the case study presents the method of searching for the most adequate manufacturing subsystem for making the order on the basis of the cost calculation. The logistics manufacturing system consisting of three subsystems is subject to thorough numerical analysis in order to determine the plant minimising manufacturing costs. The operating as well as fixed costs are taken into account. The key points responsible for excessive costs are detected. The thorough analysis based on modified operating as well as fixed costs is carried out.

\section{Mathematical model}

It is assumed that the complex logistics system consists of $\Pi$ manufacturing plants where orders can be made. Further, it is assumed that each $\pi$-th manufacturing system, $\pi=1, \ldots, \Pi$ is arranged in the same way in terms of the arrangement of machines, distances between them, production flows and all other elements which are necessary to make the production possible.

Let us assume that $N$ orders for $M$ customers are represented by the matrix of orders (1):

$$
\begin{gathered}
Z^{k}=\left[z_{m, n}^{k}\right] \\
m=1, \ldots, M, \quad n=1, \ldots, N, \quad k=1, \ldots, K
\end{gathered}
$$

where $z_{m, n}^{k}$ is the $n$-th order for the $m$-th customer at the $k$-th stage.

Let us assume that the structure of the $\pi$-th manufacturing subsystem can be defined by means of the following matrix of structure (2):

$$
\begin{gathered}
E^{\pi}=\left[e_{i, j}^{\pi}\right] \\
i=1, \ldots, I, \quad j=1, \ldots, J, \quad \pi=1, \ldots, \Pi
\end{gathered}
$$

where $e_{i, j}^{\pi}$ is the machine placed in the $i$-th row of the $j$-th column in the $\pi$-th manufacturing plant. 
The elements of the above matrix take the following values:

$$
e_{i, j}^{\pi}=\left\{\begin{array}{l}
\begin{array}{l}
\text { if the machine in the } i \text {-th row of } \\
\text { the } j \text {-th column in the } \pi \text {-th }
\end{array} \\
1 \text { manufacturing plant exists and is } \\
\text { equipped with a tool able to } \\
\text { perform a predefined operation, } \\
0 \text { otherwise. }
\end{array}\right.
$$

It is assumed that each manufacturing machine marked as $e_{i, j}^{\pi}$ equipped with its dedicated tool can perform the same operation on the $n$-th product.

Let us introduce the matrix of buffer stores in the $\pi$-th manufacturing subsystem (3):

$$
\begin{gathered}
B^{\pi}=\left[b_{i, j}^{\pi}\right] \\
i=1, \ldots, I, \quad j=1, \ldots, J, \quad \pi=1, \ldots, \Pi
\end{gathered}
$$

where $b_{i, j}^{\pi}$ is the buffer store behind the machine placed in the $i$-th row of the $j$-th column in the $\pi$-th manufacturing plant.

The element of the matrix of buffer stores takes the following values:

$$
b_{i, j}^{\pi}=\left\{\begin{array}{l}
\begin{array}{l}
\text { if the buffer store behind the } \\
\text { machine in the } i \text {-th row of } \\
\text { the } j \text {-th column in the } \pi \text {-th } \\
\text { manufacturing plant exists }
\end{array} \\
-1 \text { otherwise. }
\end{array}\right.
$$

At the same time:

$b_{i, 0}^{\pi}$ - the entrance buffer store for the machine placed before the first column of the $i$-th row in the $\pi$-th manufacturing plant;

$b_{i, J}^{\pi}$ - the final buffer store placed behind the machine in the last column of the $i$-th row in the $\pi$-th manufacturing plant.

The capacities of buffer stores are shown in the capacity matrix of buffer stores (4):

$$
\begin{gathered}
Y=\left[v\left(b_{i, j}^{\pi}\right)_{n}\right] \\
i=1, \ldots, I, j=1, \ldots, J, \pi=1, \ldots, \Pi, n=1, \ldots, N
\end{gathered}
$$

where $v\left(b_{i, j}^{\pi}\right)_{n}$ is the capacity of the buffer store placed behind the $j$-th column in the $i$-th row in case of making the $n$-th product in the $\pi$-th manufacturing plant (expressed in pieces). It is assumed that the route of machines and buffer stores in case of manufacturing the $n$-th product is identical in each $\pi$-th manufacturing plant, however, the transport time of the product from $e_{i, j}^{\pi}$ to $e_{i^{\prime}, j^{\prime}}^{\pi}$ through the $b_{i, j}^{\pi}$ (on condition it exists), $j<j^{\prime}$ may differ. Moreover, these times integrate in themselves transport times of products to a buffer store, manipulation times in the buffer stores, storing times in buffer stores and transporting products to the subsequent machine.

The length of time semi-products are stored for in buffer stores depends on the course of manufacturing and is added to the total manufacturing time.

The matrix of manufacturing routes is the same in each $\pi$-th manufacturing plant and takes the following form (5):

$$
\begin{gathered}
D=\left[d_{j, n}^{\eta}\right] \\
j=1, \ldots, J, \quad n=1, \ldots, N, \quad \eta=1, \ldots, H
\end{gathered}
$$

where $d_{j, n}^{\eta}$ is the number of the $i$-th row the $j$-th column with the machine dedicated for making the $n$-th product in the $\eta$-th step of the manufacturing process.

Actually, $d_{i, n}^{\eta}=i$ if the operation is carried out in it and $d_{j, n}^{\eta}=-i$ otherwise.

The matrix of routes of buffer stores is the same in each $\pi$-th manufacturing plant and takes the following form (6):

$$
\begin{gathered}
B=\left[b_{j, n}^{\eta}\right] \\
j=1, \ldots, J, \quad n=1, \ldots, N, \quad \eta=1, \ldots, H
\end{gathered}
$$

where $b_{j, n}^{\eta}$ is the number of the $i$-th row of the $j$-th column of the buffer store dedicated for storing the $n$-th product after the $\eta$-th stage of the manufacturing process is carried out.

Actually, $b_{j, n}^{\eta}=i$ if the buffer store is used for storing the $n$-th product and $b_{j, n}^{\eta}=-i$ if it either does not exist or is not used for storing the $n$-th product or is not necessary in the manufacturing system any more. It is assumed that the FIFO method is implemented for storing semi-products in each buffer store.

Let us define the life matrix of tools in machines in the manufacturing system (7): 


$$
\begin{gathered}
G=\left[g_{i, j}\right] \\
i=1, \ldots, I, \quad j=1, \ldots, J
\end{gathered}
$$

where $g_{i, j}$ is the life of the tool placed in the machine in the $i$-th row of its $j$-th column (expressed in conventional time units).

$$
p(\pi)_{i, j}^{k}=g_{i, j}-s(\pi)_{i, j}^{k}
$$

Let us introduce the matrix of transporting times of the $n$-th element in the $\pi$-th manufacturing system during the production process (11):

$$
\begin{aligned}
& T(\pi)_{t r}^{n}=\left[\begin{array}{ccc}
\tau(\pi)_{W \rightarrow b_{i, 0}}^{n} & \tau(\pi)_{e_{i, j} \rightarrow b_{i, 0}}^{n} & \tau(\pi)_{e_{I, J} \rightarrow b_{I, J}}^{n} \\
b_{i, 0} \rightarrow e_{i, 1} \\
W \rightarrow e_{i, 1} & b_{i, j} \rightarrow e_{i^{\prime}, j^{\prime}} \\
e_{i, j} \rightarrow e_{i^{\prime}, j^{\prime}} \rightarrow Z & e_{I, J} \rightarrow Z
\end{array}\right] \\
& i=0,1, \ldots, I, j=0,1, \ldots, J, j<j^{\prime}, n=1, \ldots, N, \pi=1, \ldots, \Pi
\end{aligned}
$$

Let us define the state matrix of tools in machines in the $\pi$-th manufacturing subsystem (8):

$$
\begin{gathered}
S(\pi)^{k}=\left[s(\pi)_{i, j}^{k}\right] \\
i=1, \ldots, I, j=1, \ldots, J, \pi=1, \ldots, \Pi, k=1, \ldots, K
\end{gathered}
$$

where $s(\pi)_{i, j}^{k}$ is the state of the tool placed in the machine in the $i$-th row of its $j$-th column in the $\pi$-th manufacturing plant at the $k$-th stage (expressed in conventional time units).

Let us define the flow capacity matrix of tools in machines in the $\pi$-th manufacturing subsystem (9):

$$
\begin{gathered}
P(\pi)^{k}=\left[p(\pi)_{i, j}^{k}\right] \\
i=1, \ldots, I, j=1, \ldots, J, \pi=1, \ldots, \Pi, k=1, \ldots, K
\end{gathered}
$$

where $p(\pi)_{i, j}^{k}$ is the flow capacity of the tool placed in the machine in the $i$-th row of the $j$-th column in the $\pi$-th manufacturing plant at the $k$-th stage (expressed in conventional time units).

Consequently, the flow capacity of the tool placed in the machine in the $i$-th row of the $j$-th column in the $\pi$-th manufacturing plant at the $k$-th stage is calculated as follows (10): where

$$
\tau(\pi)_{\begin{array}{c}
e_{i, j} \rightarrow b_{i, 0} \\
b_{i, j} \rightarrow e_{i^{\prime}, j^{\prime}} \\
e_{i, j} \rightarrow e_{i^{\prime}, j^{\prime}}
\end{array}}
$$

is the time of transporting the $n$-th product to its buffer store $\left(e_{i, j} \rightarrow b_{i, j}\right)$, from the buffer store to the subsequent machine $\left(b_{i, j} \rightarrow e_{i^{\prime}, j^{\prime}}\right)$ or directly to the subsequent machine if there is no buffer store for this operation $\left(e_{i, j} \rightarrow e_{i^{\prime}, j^{\prime}}\right)$

$$
\tau(\pi)_{\begin{array}{l}
W \rightarrow b_{i, 0} \\
b_{i, 0} \rightarrow e_{i, 1} \\
W \rightarrow e_{i, 1}
\end{array}}
$$

is the time of transporting the $n$-th product from the store of charges to the first buffer store $\left(W \rightarrow b_{i, 0}\right)$, from the first buffer store to the first machine $\left(b_{i, 0} \rightarrow e_{i, 1}\right)$ or directly to the first machine $\left(W \rightarrow e_{i, 1}\right)$,

$$
\begin{gathered}
\tau(\pi)_{e_{I, J} \rightarrow b_{I, J}}^{n} b_{I, J} \rightarrow Z \\
e_{I, J} \rightarrow Z
\end{gathered}
$$

the time of transporting the $n$-th product to the final buffer store $\left(e_{I, J} \rightarrow b_{I, J}\right)$, from the buffer store to the matrix of orders $\left(b_{I, J} \rightarrow Z\right)$ or directly to the matrix of orders $\left(e_{I, J} \rightarrow Z\right)$.

Let us introduce the matrix of manufacturing times (12):

$$
T(\pi)_{p r}^{n}=\left[\tau(\pi)_{i, j}^{p r(n)}\right]
$$


where $\tau(\pi)_{i j}^{p r(n)}$ is the manufacturing time of the $n$-th product in the machine placed in the $i$-th row of the $j$-th column in the $\pi$-th plant.

Let us introduce the matrix of replacement times of tools (13):

$$
T(\pi)_{r e p l}=\left[\tau(\pi)_{i, j}^{r e p l}\right]
$$

where $\tau(\pi)_{i, j}^{r e p l}$ is the replacement time of the tool in the machine placed in the $i$-th row of the $j$-th column in the $\pi$-th manufacturing plant.

It is assumed that there is a sufficient number of tools for the replacement process.

Let us introduce the matrix of maintenance times (14):

$$
T(\pi)_{\text {main }}=\left[\tau(\pi)_{i, j}^{\text {main }}\right]
$$

where $\tau(\pi)_{i, j}^{\text {main }}$ is the maintenance time of the machine placed in the $i$-th row of the $j$-th column in the $\pi$-th manufacturing plant.

It is assumed that maintenance times of machines placed in the $\pi$-th manufacturing plant differ.

It is assumed that the $\pi$-th manufacturing system is located in an area which determines its operating costs to a greater extent as they depend on the local dependencies i.e. labour force costs, energy costs, transport prices, taxes, infrastructure maintenance costs and other vital cost components. These costs may vary considerably, e.g. from year to year, as additional factors have to be taken into account such as the influence of political decisions, extreme weather conditions, etc.

Let us introduce the matrix of transport costs (15):

the buffer store to the subsequent machine $\left(b_{i, j}\right.$ $\left.\rightarrow e_{i^{\prime}, j^{\prime}}\right)$ or directly to the subsequent machine if there is no buffer store for this operation $\left(e_{i, j}\right.$ $\left.\rightarrow e_{i^{\prime}, j^{\prime}}\right)$,

$$
\begin{aligned}
c(\pi)_{W \rightarrow b_{i, 0}}^{\operatorname{tr}(n)} \\
b_{i, 0} \rightarrow e_{i, 1} \\
W \rightarrow e_{i, 1}
\end{aligned}
$$

the cost of transporting the $n$-th product in the $\pi$-th plant from the store of charges to the first buffer store $\left(W \rightarrow b_{i, 0}\right)$, from the first buffer store to the first machine $\left(b_{i, 0} \rightarrow e_{i, 1}\right)$ or directly to the first machine $\left(W \rightarrow e_{i, 0}\right.$ ),

$$
c(\pi)_{\substack{t r(n) \\ e_{I, J} \rightarrow b_{I, J} \\ b_{I, J} \rightarrow Z \\ e_{I, J} \rightarrow Z}}
$$

the cost of transporting the $n$-th product in the $\pi$-th plant to the last buffer store $\left(e_{I, J} \rightarrow b_{I, J}\right)$, from the last buffer store to the matrix of orders $\left(b_{I, J} \rightarrow Z\right)$ or directly to the matrix of orders $\left(e_{I, J}\right.$ $\rightarrow Z)$

Let us introduce the matrix of production costs (16):

$$
C(\pi)^{p r}=\left[c(\pi)_{i, j}^{p r(n)}\right]
$$

where $c(\pi)_{i, j}^{p r(n)}$ the production cost of the $n$-th product made in the machine placed in the $i$-th row of the $j$-th column in the $\pi$-th plant.

The matrix of costs of storing charge materials takes the following form (17):

$$
C(\pi)_{s t}^{w}=\left[c(\pi)_{w, n}^{s t}\right]
$$

$$
\begin{aligned}
& C(\pi)^{\operatorname{tr}}=\left[c(\pi)^{\operatorname{tr}(n)}\right]=\left[\begin{array}{ccc}
c(\pi)_{W \rightarrow b_{i, 0}}^{\operatorname{tr}(n)} & c(\pi)_{e_{i, j} \rightarrow b_{i, j}}^{\operatorname{tr}(n)} & c(\pi)_{\substack{e_{I, J} \rightarrow b_{I, J} \\
b_{i, j} \rightarrow e^{\prime} j^{\prime} \\
b_{I, J} \rightarrow e_{i, 1} \\
W \rightarrow e_{i, 1} \\
e_{i, j} \rightarrow e_{i^{\prime}, j^{\prime}}}}^{e_{I, J} \rightarrow Z}
\end{array}\right] \\
& i=0,1, \ldots, I, j=0,1, \ldots, J, j<j^{\prime}, n=1, \ldots, N, \pi=1, \ldots, \Pi
\end{aligned}
$$

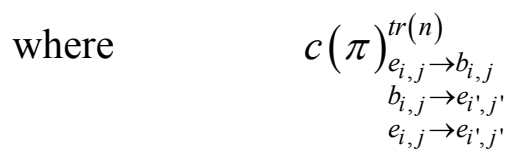

is the cost of transporting the $n$-th product in the $\pi$-th plant to its buffer store $\left(e_{i, j} \rightarrow b_{i, j}\right)$, from where $c(\pi)_{w, n}^{s t}$ is the storing cost of the $w$-th charge material for making the $n$-th product in the machine placed in the $\pi$-th plant.

The matrix of costs of storing semi-products in the buffer stores takes the following form (18): 


$$
C(\pi)^{b u f_{-} s t}=\left[c(\pi)_{m, n}^{b u f_{-} s t}\right]
$$

where $c(\pi)_{m, n}^{b u f_{-} s t}$ is the storing cost of the $n$-th semi-product made for the $m$-th customer in the $\pi$-th plant in the buffer store.

The matrix of costs of storing ready products takes the following form (19):

$$
C(\pi)^{s t_{-} z}=\left[c(\pi)_{m, n}^{s t_{-} z}\right]
$$

where $c(\pi)_{m, n}^{s t z z}$ is the storing cost of the $n$-th ready product made for the $m$-th customer in the ready product store in the $\pi$-th manufacturing plant.

The matrix of maintenance costs takes the following form (20):

$$
C(\pi)^{\text {main }}=\left[c(\pi)_{i, j}^{\text {main }}\right]
$$

where $c(\pi)_{i, j}^{\text {main }}$ the maintenance cost of the machine placed in the $i$-th row of the $j$-th column in the $\pi$-th manufacturing plant (per one unit of the order made in this machine).

The matrix of replacement costs of tools takes the following form (21):

$$
C(\pi)^{r e p l}=\left[c(\pi)_{i, j}^{r e p l}\right]
$$

where $c(\pi)_{i, j}^{r e p l}$ is the replacement cost of the tools in the machine placed in the $i$-th row of the $j$-th column in the $\pi$-th manufacturing plant (per one unit of the order made in this machine).

The vector of standstill costs takes the following form (22):

$$
C(\pi)^{\text {still }}=\left[c(\pi)^{\text {still }}\right]
$$

where $c(\pi)^{\text {still }}$ is the unit standstill costs of the $\pi$-th manufacturing plant (per one unit of the order matrix).

The vector of costs of implementing heuristic algorithms takes the following form (23):

$$
C(\pi)_{\alpha}=\left[c(\pi)_{\chi}\right]
$$

where $c(\pi)_{\chi}$ is the cost of implementing the $\chi$-th heuristic algorithm in the $\pi$-th manufacturing plant, $\alpha \leq \chi \leq A$.
The vector of hidden operating costs takes the following form (24):

$$
C(\pi)^{h c}=\left[c(\pi)^{h c}\right]
$$

where $c(\pi)^{h c}$ is the hidden unit cost of the $\pi$-th manufacturing plant (per one unit of the order matrix).

The total predicted costs of the 1 -th sub-company in case of making the order represented by $Z^{0}$ are calculated as follows (25):

$$
\begin{aligned}
C_{\text {total }}^{\pi}= & c(\pi)^{t r(n)}+c(\pi)_{i, j}^{p r(n)}+c(\pi)_{w, n}^{s t} \\
& +c(\pi)_{m, n}^{b u f_{s} s t}+c(\pi)_{m, n}^{s t \_z} \\
& +\left(\sum_{j=1}^{J} \sum_{i=1}^{I} c(\pi)_{i, j}^{\text {main }}+\sum_{j=1}^{J} \sum_{i=1}^{I} c(\pi)_{i, j}^{r e p l}\right. \\
& \left.+c(\pi)^{\text {still }}+\sum_{1 \leq \chi \leq A} c(\pi)_{\chi}+c(\pi)^{h c}\right) \\
& \cdot \sum_{n=1}^{N} \sum_{m=1}^{M} z_{m, n}^{0}
\end{aligned}
$$

\section{Manufacturing Strategies and Heuristics}

To control the manufacture of customers' orders there is a need to assume that the chosen manufacturing strategy along with the heuristic algorithm decide how and in which available manufacturing plant to make an order matrix as one of the goals of the paper is to present the method of choosing the subsystem which minimises the total costs. To begin the simulation process, there is a need to choose a manufacturing strategy according to which the order matrix elements should be made. It is assumed that orders can be made in the $\pi$-th manufacturing plant, $\pi=1, \ldots, \Pi$. The adjustment of the $n$-th order of the $\mathrm{m}$-th customer to the $\pi$-th manufacturing plant is expressed by the following adjustment matrix (26):

$$
\begin{gathered}
\Xi=\left[\xi_{(m, n) \rightarrow \pi}\right] \\
m=1, \ldots, M, n=1, \ldots, N, \pi=1, \ldots, \Pi
\end{gathered}
$$


where $\xi_{(m, n) \rightarrow \pi}$ is adjustment of the $n$-th order of the $m$-th customer to the $\pi$-th manufacturing plant.

At the same time elements of this matrix take the following values:

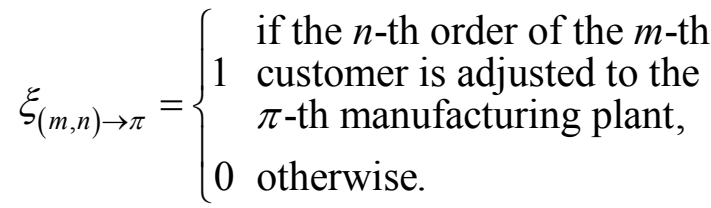

On some occasions it is reasonable to make orders in a manufacturing plant situated closer to customers to minimise the final product cost by minimising transport costs. To solve this problem from a mathematical modelling perspective, it is necessary to introduce the matrix responsible for choosing the adequate manufacturing plant for the $n$-th order of the $m$-th customer on the basis of the chosen criterion. As an example, the distance criterion can be decisive in case of choosing the right manufacturing plant.

Let us introduce the distance matrix which is responsible for choosing the adequate plant (27):

$$
\begin{gathered}
\Delta=\left[\delta_{m \rightarrow n}\right] \\
m=1, \ldots, M, \pi=1, \ldots, \Pi
\end{gathered}
$$

where $\delta_{m \rightarrow \pi}$ is the distance from the $m$-th customer to the $\pi$-th manufacturing plant (expressed in distance units).

At the same time if $\delta_{m \rightarrow \pi}=-1$, then it is not possible to match the $m$-th customer's order with the $\pi$-th manufacturing plant for some reasons (e.g. the lack of transport means, natural barriers, business conflicts, etc.). Moreover, it is obvious that each strategy should be chosen depending on the costs it generates.

Manufacturing strategies are unavoidably associated with control heuristic algorithms which are responsible for making a decision about choosing the manufacturing plant from the available ones. Control of the discused logistics manufacturing system requires implementing adequate methods which can be grouped as follows:

1. Algorithm $\alpha_{1}$ chooses the nearest $\pi$-th manufacturing plant for the $m$-th customer's order.
2. Algorithm $\alpha_{2}$ chooses the biggest $n$-th order which is located the nearest to the $\pi$-th manufacturing plant.

3. Algorithm $\alpha_{3}$ chooses the $\pi$-th manufacturing plant characterised by the lowest total state of tools in all machines for the nearest $m$-th customer.

4. Algorithm $\alpha_{4}$ chooses the $\pi$-th manufacturing plant characterised by the lowest total manufacturing costs.

5. Algorithm $\alpha_{5}$ chooses the $\pi$-th manufacturing plant characterised by the lowest total manufacturing time.

6. Algorithm $\alpha_{6}$ chooses the $\pi$-th manufacturing plant characterised by the highest total quality.

7. Algorithms $\alpha_{1}$ and $\alpha_{2}$ choose at random.

8. Algorithms $\alpha_{1}$ and $\alpha_{3}$ choose at random.

9. Algorithms $\alpha_{2}$ and $\alpha_{3}$ choose at random.

10. Algorithms $\alpha_{1}, \alpha_{2}$ and $\alpha_{3}$ choose at random.

11 . The $m$-th customer and the $\pi$-th manufacturing plant choose at random.

12 . The $n$-th order and the $\pi$-th manufacturing plant choose at random.

As the number of heuristics is big there is a need to carry out a simulation process in order to verify which one of them or which combination of heuristic algorithms is able to minimise the total manufacturing costs. Initial data are subject to change according to customers' orders, which requires checking the usefulness of certain heuristics or their configuration. This is the reason why the simulator of the logistics manufacturing system seems to be unavoidable.

\section{Simulator of the Logistics System}

Preparing a fully operational simulator of the logistics system requires meeting all the needs of the system projection phase in order to pass a functional IT product into the implementation phase. It is also essential to avoid any errors while building simulators of real systems. These errors may result in malfunctioning of the system while making the vital manufacturing decision. Modelling information logistics systems 
also requires a proper approach which means the need to analyse the real environment emerges thoroughly. This forms the basis for the specification assumptions followed by the adequate project of the system. These are then taken into account while building a mathematical model which reflects the real system. The final information model is subject to programming which results in the so-called dedicated simulator for the real system. The simulator is to be tested thoroughly and subsequently validated. When these two processes are successful, it is possible to pass the ready system to the process of implementation which can be divided into the phases of IT system deployment consisting of the pre-sale phase (promotion, presentation, license, education, distribution), post-sale phase (installation, configuration, training, simulator, integration) and operation phase (consultation, modification, service, care, development). After the software becomes unusable or unnecessary it is archived. The simulator used for carrying out the simulation process was built with the use of the iteration model shown in Figure 1.

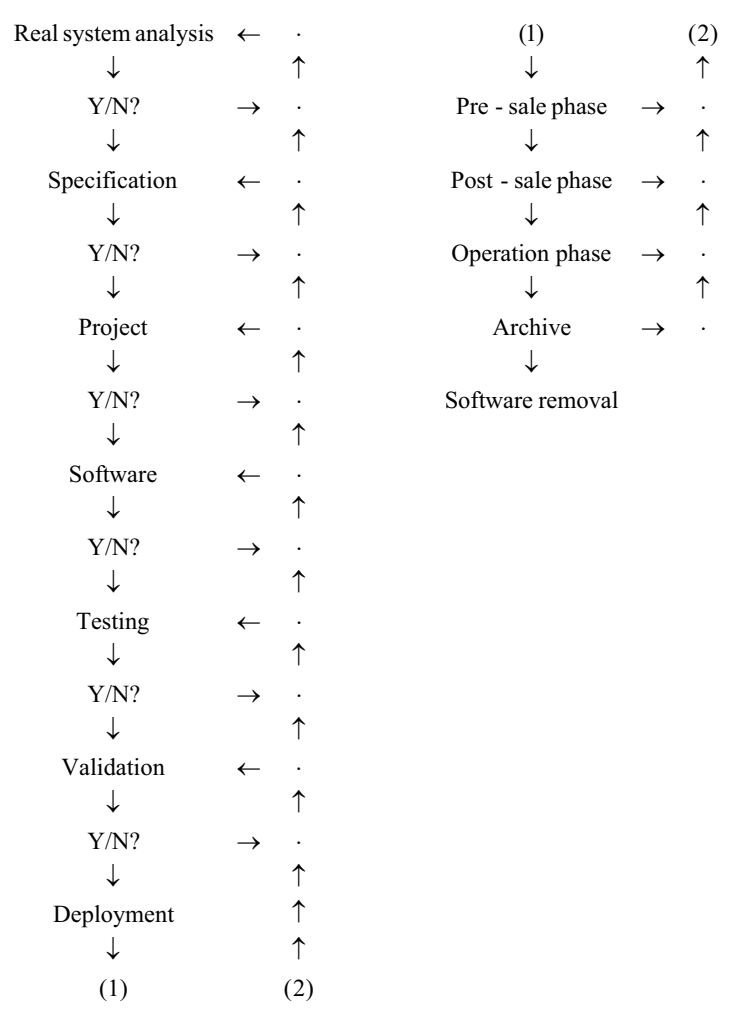

Figure 1. The iteration model for creating the simulator of the logistics system.
It is not always obvious where problems can appear during creating and implementing dedicated software. This is the reason why there should always be possibilities to return to any of the predeceasing stage. The simulator of the logistics manufacturing system was designed in the form of three integrated modules: the data entry module, the simulation module and the results module. In addition, each of the modules includes different working modes. The data entry module modes differ depending on the source of data. The data can be inputted via keyboard, generated randomly, with or without the seed value, or loaded directly from a file. The simulation module allows the operator of the system to perform a single simulation, experiments at random or with a set of heuristics as well as a step-by-step simulations. The results module allows the operator to analyse and compare results depending on the number of simulations performed, as well as on their type.

The simulator was written in the $\mathrm{C \#}$ programming language, using .NET Framework 4 and Microsoft Visual C\# 2012 Express programming environment. A DataGridFunctions library was created for the needs of the system. The library consists of two classes including the set of static methods working on arrays and DataGridView objects. It implements exception handling allowing an easier detection of incorrect input data. During the system testing the administrator and user manuals were created, including instructions for the installation process and the typical work with the system, showing its protection mechanisms against incorrect input data. There were also analysed practical examples results of which were confronted with these returned by the simulator allowing verifying the correctness of the system calculations. Testing the simulator required creating the set of initial data from the range of reasonable values based on thorough observations carried out in a few manufacturing plants. However, for the purpose of the simulation process, there was a need for the initial data simplification. The verification result was positive so all the values returned by the system may now be considered as correct.

The data for the subsequent simulation process were generated within the assumed ranges. The data generation process can be replicated without 
any limitations. Once the data are generated, it can be optionally modified in order to test alternative possibilities of making order matrix elements.

\section{Case study - Preliminary Cost Analysis}

Having created and tested the simulator of the logistics manufacturing system it became possible to carry out an illustrative manufacturing process. The initial data were generated at random from the defined range of real data. It is assumed that the complex manufacturing system consists of three subsystems which are identical, however, they are characterised by different operation and fixed costs. The goal of the simulation is to find the plant which minimises total manufacturing costs.

The study case is based on the calculation analysis of operating and fixed costs of the sample manufacturing plant from the automotive industry. Operating costs are presented in Table 1 whereas fixed costs for each $\pi$-th manufacturing subsystem are presented in Table 2 . The manufacturing subsystem characterised by the lowest manufacturing costs is searched for.
The sample matrix of the subsystems structure is proposed as follows (28):

$$
\begin{aligned}
\underset{1 \leq \pi \leq 3}{\wedge}\left(e_{1,1}^{\pi}\right. & =1 ; e_{1,2}^{\pi}=1 ; e_{1,3}^{\pi}=1 ; e_{1,4}^{\pi}=0 ; \\
e_{2,1}^{\pi} & =0 ; e_{2,2}^{\pi}=1 ; e_{2,3}^{\pi}=1 ; e_{2,4}^{\pi}=1 ; \\
e_{3,1}^{\pi} & =1 ; e_{3,2}^{\pi}=0 ; e_{3,3}^{\pi}=0 ; e_{3,4}^{\pi}=1 ; .
\end{aligned}
$$

After carrying out the simulation process according to the procedure shown in the mathematical model with the use of manufacturing strategies along with implementing heuristic algorithms, the results of the total manufacturing costs for each $\pi$-the subsystem are calculated as follows:

$$
\begin{aligned}
& C_{\text {total }}^{1}=900,8 \\
& C_{\text {total }}^{2}=905,6 \\
& C_{\text {total }}^{3}=920,4
\end{aligned}
$$

As seen in Table 1, the lowest operating costs

\begin{tabular}{|c|c|c|c|c|c|c|c|c|c|c|c|c|c|c|c|c|c|c|}
\hline Operating costs & \multicolumn{3}{|c|}{$c(\pi)^{t r(n)}$} & \multicolumn{3}{|c|}{$c(\pi)_{i, j}^{p r(n)}$} & \multicolumn{3}{|c|}{$c(\pi)_{w, n}^{s t}$} & \multicolumn{3}{|c|}{$c(\pi)_{m, n}^{b u f_{s} t}$} & \multicolumn{3}{|c|}{$c(\pi)_{m, n}^{s t z}$} & \multicolumn{3}{|c|}{$\Sigma$} \\
\hline Subsystem $\pi \rightarrow$ & 1 & 2 & 3 & 1 & 2 & 3 & 1 & 2 & 3 & 1 & 2 & 3 & 1 & 2 & 3 & 1 & 2 & 3 \\
\hline$z_{1,1}^{0}=13$ & 1 & 2 & 1 & 3 & 1 & 1 & 1 & 1 & 3 & 1 & 3 & 2 & 1 & 1 & 1 & 91 & 104 & 104 \\
\hline$z_{2,2}^{0}=15$ & 2 & 1 & 1 & 1 & 2 & 2 & 1 & 1 & 1 & 1 & 1 & 1 & 2 & 2 & 1 & 90 & 105 & 90 \\
\hline$z_{2,4}^{0}=11$ & 1 & 2 & 1 & 1 & 1 & 1 & 3 & 2 & 1 & 2 & 1 & 1 & 1 & 3 & 1 & 88 & 99 & 55 \\
\hline$z_{3,2}^{0}=5$ & 3 & 2 & 2 & 1 & 1 & 1 & 2 & 1 & 1 & 2 & 1 & 1 & 1 & 1 & 1 & 45 & 30 & 30 \\
\hline$z_{3,5}^{0}=17$ & 1 & 1 & 2 & 2 & 1 & 2 & 1 & 1 & 1 & 1 & 2 & 1 & 1 & 1 & 1 & 102 & 102 & 119 \\
\hline$z_{4,1}^{0}=7$ & 1 & 3 & 1 & 1 & 2 & 2 & 1 & 3 & 1 & 1 & 1 & 1 & 1 & 1 & 1 & 35 & 70 & 42 \\
\hline$z_{4,2}^{0}=5$ & 3 & 1 & 1 & 1 & 2 & 1 & 2 & 1 & 1 & 3 & 1 & 1 & 3 & 1 & 1 & 50 & 30 & 25 \\
\hline$z_{4,3}^{0}=10$ & 1 & 1 & 1 & 1 & 1 & 3 & 1 & 1 & 1 & 1 & 1 & 1 & 1 & 1 & 1 & 60 & 50 & 70 \\
\hline$z_{4,4}^{0}=23$ & 2 & 1 & 1 & 2 & 3 & 3 & 1 & 1 & 3 & 1 & 1 & 1 & 1 & 1 & 1 & 161 & 161 & 207 \\
\hline$z_{5,1}^{0}=12$ & 1 & 1 & 1 & 3 & 2 & 1 & 1 & 1 & 2 & 3 & 1 & 2 & 2 & 1 & 1 & 108 & 72 & 84 \\
\hline Total & & & & & & & & & & & & & & & & 830 & 823 & 826 \\
\hline
\end{tabular}
are generated for the subsystem $\pi=2$. However, Table 2 shows that the lowest fixed costs are detected in the subsystem $\pi=1$. The weakest points are diagnosed and marked in adequate

Table 1. Operating costs of the sample complex system. 
cells in Table 1. It is possible to prove that if there are certain improvements introduced leading to minimising operating times in chosen logistics areas, the total costs can be minimised. For illustration reasons, sample chosen key cells are modified, which leads to minimising the total costs (operating and fixed) as follows:

Order: $z_{3,5}^{0}=17$

Modified plant: $\quad \pi=3$

Modified cost element $c(n)^{\operatorname{tr}(\pi)}: \quad 2 \rightarrow 1$

Modified cost element $c(n)_{i, j}^{p r(\pi)}: \quad 2 \rightarrow 1$

Modified total order making

costs: $\quad C_{\text {total }}^{3 \_\bmod }=886,4$

The system is complex enough to search for cost improvements in it, either as a whole or in each $\pi$-th subsystem.

The total costs are subject to the thorough analysis which takes into account the costs shown in Table 2 i.e. fixed costs as the whole group of logistics costs and hidden costs, especially as it is assumed that these costs comprise unavoidable investments. They increase the fixed costs at the beginning, however, in the long run, the total costs decrease as shown in Table 3. The hidden cost coefficient $\varphi_{h c}$ is introduced for the analysis needs. It is meant to include the problem of investment improvements as well. It is

Table 2. Fixed unit costs of the sample complex system.

\begin{tabular}{|c||c|c|c|}
\hline \multicolumn{1}{|c||}{\multirow{2}{*}{ Group of costs }} & \multicolumn{3}{c|}{ Unit costs } \\
\cline { 2 - 4 } & $\pi=1$ & $\pi=2$ & $\pi=3$ \\
\hline \hline$\sum_{j=1}^{J} \sum_{i=1}^{I} c(\pi)_{i, j}^{\text {main }}$ & 0,1 & 0,1 & 0,2 \\
\hline$\sum_{j=1}^{J} \sum_{i=1}^{I} c(\pi)_{i, j}^{r e p l}$ & 0,2 & 0,1 & 0,1 \\
\hline$c(\pi)^{\text {still }}$ & 0,1 & 0,2 & 0,1 \\
\hline$\sum_{1 \leq \chi \leq A} c(\pi)_{\chi}$ & 0,1 & 0,1 & 0,3 \\
\hline$c(\pi)^{h c}$ & 0,1 & 0,2 & 0,1 \\
\hline Sum of fixed unit costs & 0,6 & 0,7 & 0,8 \\
\hline Total & $\mathbf{7 0 , 8}$ & $\mathbf{8 2 , 6}$ & $\mathbf{9 4 , 4}$ \\
\hline
\end{tabular}

assumed that in case of $\varphi_{h c}=1$ there is not any decrease in hidden costs and they are subject to modifying in the 1 st period only. It is justified by the fact of implementing lean methods fully once, which means there is not any real need for further amendments in subsequent periods.

The fixed cost coefficient $\varphi_{f_{c}}$ is introduced for the analysis needs. This coefficient assumes that there can be decrease in fixed costs if lean methods are implemented.

The period coefficient $\varphi_{\text {per }}$ is introduced in order to determine how much the passage of time influences the total costs.

The difference coefficient $\varphi_{\text {dif }}$ illustrates the influence of implementing procedures leading to decreasing fixed costs on the subsystem total costs.

The values shown in Table 3 are then calculated on the basis of the following formula (29):

$$
\begin{aligned}
C_{\text {total }}^{\pi} & =\varphi_{\text {per }} \cdot\left(c(\pi)^{t r(n)}+c(\pi)_{i, j}^{p r(n)}+c(\pi)_{w, n}^{s t}\right. \\
& +c(\pi)_{m, n}^{b u f_{-} s t}+c(\pi)_{m, n}^{s t \_z} \\
& +\varphi_{f c}\left(\sum_{j=1}^{J} \sum_{i=1}^{I} c(\pi)_{i, j}^{\text {main }}+\sum_{j=1}^{J} \sum_{i=1}^{I} c(\pi)_{i, j}^{r e p l}\right. \\
& \left.+c(\pi)^{\text {still }}+\sum_{1 \leq \chi \leq A} c(\pi)_{\chi}+\varphi_{h c} \cdot c(\pi)^{h c}\right)
\end{aligned}
$$

$$
\left.\cdot \sum_{n=1}^{N} \sum_{m=1}^{M} z_{m, n}^{0}\right)
$$

As seen in Table 3, in case of $\varphi_{f c}=1, \varphi_{h c}=2$ and $\varphi_{\text {per } 5}=0,96, C_{\text {total }}^{1}=900,8$ which is the best result conquered by 5,89 cost units. However, in case of $\varphi_{f_{c}}=0,95, \varphi_{h c}=1$ and $\varphi_{\text {per } 5}=0,96$ this result is conquered by 0,23 cost unit.

The total modified costs for $\pi=3$ are presented in Figure 2. As seen in Figure 2, total modified costs for $\pi=3$ decrease with the passage of time. Due to the assumptions that fixed costs can be decreased maximally by $10 \%$, there are 5 time intervals. In this case the total modified costs are shown as the function of the fixed costs and the period coefficient. Moreover, it is assumed that fixed costs cannot be decreased any further. 
Table 3. Analysis of total costs for $\pi=3$.

\begin{tabular}{|c|c|c|c|c|c|c|c|c|c|c|c|c|}
\hline \multirow[b]{2}{*}{ No. } & \multirow[b]{2}{*}{$\varphi_{f c}$} & \multicolumn{3}{|c|}{$\begin{array}{c}\text { 1st period } \\
\varphi_{\text {per_1 }}=1\end{array}$} & \multicolumn{2}{|c|}{$\begin{array}{c}\text { 2nd period } \\
\varphi_{p e r_{-} 2}=0,99\end{array}$} & \multicolumn{2}{|c|}{$\begin{array}{c}\text { 3rd period } \\
\varphi_{\text {per_-3 }}=0,98\end{array}$} & \multicolumn{2}{|c|}{$\begin{array}{c}\text { 4th period } \\
\varphi_{\text {per-4 }}=0,97\end{array}$} & \multicolumn{2}{|c|}{$\begin{array}{c}\text { 5th period } \\
\varphi_{\text {per_-5 }}=\mathbf{0 , 9 6}\end{array}$} \\
\hline & & $\varphi_{h c}=1$ & $\varphi_{h c}=2$ & $\varphi_{h c}=3$ & $\varphi_{h c}=2$ & $\varphi_{h c}=3$ & $\varphi_{h c}=2$ & $\varphi_{h c}=3$ & $\varphi_{h c}=2$ & $\varphi_{h c}=3$ & $\varphi_{h c}=2$ & $\varphi_{h c}=3$ \\
\hline 1 & 1,00 & 920,40 & 932,20 & 944,00 & 922,88 & 934,56 & 913,56 & 925,12 & 904,23 & 915,68 & 894,91 & 906,24 \\
\hline 2 & 0,99 & 9,46 & 931,14 & 942,82 & 921,83 & 933,39 & 912,52 & 923,96 & 903,20 & 914,53 & 893, & 905,10 \\
\hline 3 & 0,98 & 918,51 & 930,08 & 941,64 & 920,78 & 932,22 & 911,48 & 922,81 & 902,17 & 913,39 & 892,87 & 903,97 \\
\hline 4 & 0,97 & 917,57 & 929,01 & 940,46 & 919,72 & 931,06 & 910,43 & 921,65 & 901,14 & 912,24 & 891,85 & 902,84 \\
\hline 5 & 0,96 & 916,62 & 927,75 & 939,28 & 918,67 & 929,89 & 909,39 & 920,49 & 900,11 & 911,10 & 890,83 & 901,70 \\
\hline 6 & 0,95 & 915,68 & 926,89 & 938,10 & 917,62 & 928,72 & 908,35 & 919,34 & 899,08 & 909,95 & 889,81 & 900,57 \\
\hline 7 & 0,94 & 914,74 & 925,83 & 936,92 & 916,57 & 927,55 & 907,31 & 918,18 & 898,05 & 908,81 & 888,79 & 899,44 \\
\hline 8 & 0,93 & 913,79 & 924,77 & 935,74 & 915,52 & 926,38 & 906,27 & 917,03 & 897,02 & 907,66 & 887,77 & 898,31 \\
\hline 9 & 0,92 & 912,85 & 923,70 & 934,56 & 914,47 & 925,21 & 905,23 & 915,87 & 895,99 & 906,52 & 886,75 & 897,17 \\
\hline 10 & 0,91 & 911,90 & 922,64 & 933,38 & 913,42 & 924,05 & 904,19 & 914,71 & 894,96 & 905,37 & 885,73 & 896,04 \\
\hline 11 & 0,90 & 910,96 & 921,58 & 932,20 & 912,36 & 922,88 & 903,15 & 913,56 & 893,93 & 904,23 & 884,71 & 894,91 \\
\hline \multicolumn{2}{|c|}{$\varphi_{d i f}$} & 9,44 & 10,62 & 11,80 & 10,51 & 11,68 & 10,41 & 11,56 & 10,30 & 11,45 & 10,19 & 11,33 \\
\hline
\end{tabular}

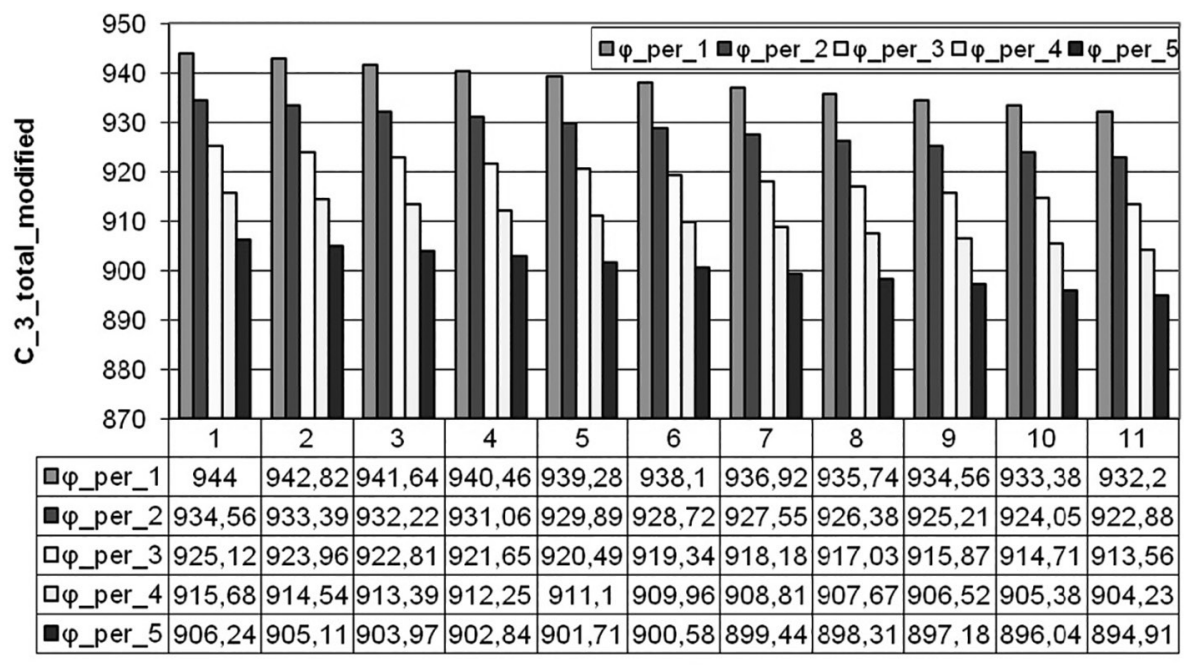

$\varphi \_$fc

Figure 2. Total modified costs for $\pi=3$.

The case study is based on the specific mathematical model and requires following all its requirements. Final decision about the best manufacturing subsystem depends on many factors such as initial data (orders, fixed and operation costs), manufacturing times of orders, etc. It is obvious that an identical system equipped with other initial data will generate results which may differ from the ones obtained hereby. This is the main reason why manufacturing systems are to be treated as autonomous ones, which requires an independent approach to finding a satisfactory solution for each of them. 
Table 4. List of symbols.

\begin{tabular}{|c|c|}
\hline$B^{\pi}$ & the matrix of buffer stores in the $\pi$-th manufacturing subsystem \\
\hline$b_{i, j}^{\pi}$ & $\begin{array}{l}\text { the buffer store placed behind the machine placed in the } i \text {-th row of the } j \text {-th column in the } \pi \text {-th } \\
\text { manufacturing }\end{array}$ \\
\hline$b_{i, 0}^{\pi}$ & $\begin{array}{l}\text { the entrance buffer store for the machine placed before the first column of the } i \text {-th row in the } \pi \text {-th } \\
\text { manufacturing plant }\end{array}$ \\
\hline$b_{i, J}^{\pi}$ & $\begin{array}{l}\text { the final buffer store placed behind the machine in the last column of the } i \text {-th row in the } \pi \text {-th } \\
\text { manufacturing plant }\end{array}$ \\
\hline$B$ & the matrix of routes of buffer stores \\
\hline$b_{j, n}^{\eta}$ & $\begin{array}{c}\text { the number of the } i \text {-th row the } j \text {-th column of the buffer store dedicated for storing the } n \text {-th product } \\
\text { after the } \eta \text {-th step of the manufacturing process }\end{array}$ \\
\hline$c(\pi)_{\chi}$ & the cost of implementing $\chi$-th heuristic algorithm in the the $\pi$-th manufacturing plant \\
\hline$c(\pi)_{\alpha}$ & the vector of costs of implementing heuristic algorithms \\
\hline$c(\pi)_{m, n}^{b u f s t}$ & the storing cost of the $n$-th semi-product in the buffer stores made for the $m$-th customer in the $\pi$-th plant \\
\hline$c(\pi)^{b u f_{-} s t}$ & the matrix of costs of storing semi-products in the buffer stores \\
\hline$c(\pi)^{h c}$ & the unit hidden costs of the $\pi$-th manufacturing plant \\
\hline$C(\pi)^{h c}$ & the vector of hidden operating costs \\
\hline$c(\pi)_{i, j}^{\text {main }}$ & $\begin{array}{l}\text { the maintenance cost of the machine placed in the } i \text {-th row of the } j \text {-th column in the } \pi \text {-th } \\
\text { manufacturing plant }\end{array}$ \\
\hline$C(\pi)^{\text {main }}$ & the matrix of maintenance costs \\
\hline$c(\pi)_{i, j}^{p r(n)}$ & $\begin{array}{l}\text { the production cost of the } n \text {-th product made in the machine placed in the } i \text {-th row of the } j \text {-th column } \\
\text { in the } \pi \text {-th plant }\end{array}$ \\
\hline$c(\pi)^{p r}$ & the matrix of production costs \\
\hline$c(\pi)_{i, j}^{r e p l}$ & $\begin{array}{l}\text { the replacement cost of the tools in the machine placed in the } i \text {-th row of the } j \text {-th column in the } \pi \text {-th } \\
\text { manufacturing plant }\end{array}$ \\
\hline$C(\pi)^{r e p l}$ & the matrix of replacement costs of tools \\
\hline$c(\pi)_{w, n}^{s t}$ & $\begin{array}{l}\text { the storing cost of the } w \text {-th charge material for making the } n \text {-th product in the machine placed in the } \\
\qquad \pi \text {-th plant }\end{array}$ \\
\hline$c(\pi)_{s t}^{w}$ & the matrix of costs of storing charge materials \\
\hline$c(\pi)_{m, n}^{s t z z}$ & $\begin{array}{l}\text { the storing cost of the } n \text {-th ready product made for the m-th customer in the ready product store in the } \\
\pi \text {-th manufacturing plant }\end{array}$ \\
\hline$c(\pi)^{s t z}$ & the matrix of costs of storing ready products \\
\hline$c(\pi)^{\text {still }}$ & the unit standstill costs of the $\pi$-th manufacturing plant \\
\hline$C(\pi)^{\text {still }}$ & the vector of standstill costs \\
\hline$c(\pi)_{e_{i, j} \rightarrow b_{i, j}}^{\operatorname{tr}(n)} \mid$\begin{tabular}{r|}
$b_{i, j} \rightarrow e_{i^{\prime}, j^{\prime}}$ \\
$e_{i, j,} \rightarrow e_{i^{\prime}, j^{\prime}}$
\end{tabular} & $\begin{array}{c}\text { the cost of transporting the } n \text {-th product in the } \pi \text {-th plant to its buffer store }\left(e_{i, j} \rightarrow e_{i^{\prime}, j^{\prime}}\right) \text {, from the buffer } \\
\text { store to the subsequent machine }\left(b_{i, j} \rightarrow e_{i^{\prime}, j^{\prime}}\right) \text { or directly to the subsequent machine if there is no buffer } \\
\text { store for this operation }\left(e_{i, j} \rightarrow e_{i^{\prime}, j^{\prime}}\right)\end{array}$ \\
\hline 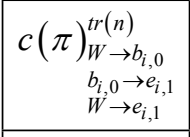 & $\begin{array}{l}\text { the cost of transporting the } n \text {-th product in the } \pi \text {-th plant from the store of charges to the first buffer } \\
\text { store }\left(W \rightarrow b_{i, 0}\right) \text {, from the first buffer store to the first machine }\left(b_{i, 0} \rightarrow e_{i, 1}\right) \text { or directly to the first } \\
\text { machine }\left(W \rightarrow e_{i, 1}\right)\end{array}$ \\
\hline 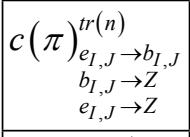 & $\begin{array}{l}\text { the cost of transporting the } n \text {-th product in the } \pi \text {-th plant to the last buffer store }\left(e_{I, J} \rightarrow b_{I, J}\right) \text {, from the } \\
\text { buffer store to the matrix of orders }\left(b_{I, J} \rightarrow Z\right) \text { or directly to the matrix of orders }\left(e_{I, J} \rightarrow Z\right)\end{array}$ \\
\hline$C(\pi)^{t r}$ & the matrix of transport costs \\
\hline$D$ & the matrix of manufacturing routes \\
\hline$d_{j, n}^{\eta}$ & $\begin{array}{l}\text { the number of the } i \text {-th row the } j \text {-th column with the machine dedicated for making the } n \text {-th product in } \\
\text { the } \eta \text {-th step of the manufacturing process }\end{array}$ \\
\hline
\end{tabular}


Table 4. List of symbols.

\begin{tabular}{|c|c|}
\hline$E^{\pi}$ & the $\pi$-th manufacturing subsystem \\
\hline$e_{i, j}^{\pi}$ & the machine placed in the $i$-th row of the $j$-th column in the $\pi$-th manufacturing plant \\
\hline$G$ & the life matrix of tools in machines in the manufacturing system \\
\hline$g_{i, j}$ & $\begin{array}{l}\text { the life of the tool placed in the machine in the } i \text {-th row of its } j \text {-th column } \\
\text { (expressed in conventional time units) }\end{array}$ \\
\hline$P(\pi)^{k}$ & the flow capacity matrix of tools in machines in the $\pi$-th manufacturing subsystem \\
\hline$p(\pi)_{i, j}^{k}$ & $\begin{array}{l}\text { the flow capacity of the tool placed in the machine in the } i \text {-th row of its } j \text {-th column in the } \pi \text {-th } \\
\text { manufacturing plant at the } k \text {-th stage }\end{array}$ \\
\hline$s(\pi)_{i, j}^{k}$ & $\begin{array}{l}\text { the state of the tool placed in the machine in the } i \text {-th row of its } j \text {-th column in the } \pi \text {-th manufacturing } \\
\text { plant at the } k \text {-th stage }\end{array}$ \\
\hline$S(\pi)^{k}$ & the state matrix of tools in machines in the $\pi$-th manufacturing system at the $k$-th stage \\
\hline$\tau(\pi)_{i, j}^{r e p l}$ & $\begin{array}{l}\text { the maintenance time of the machine placed in the } i \text {-th row of the } j \text {-th column in } \pi \text {-th } \\
\text { manufacturing plant }\end{array}$ \\
\hline$T(\pi)_{\text {main }}$ & the matrix of maintenance times of machines in the $\pi$-th manufacturing plant \\
\hline$\tau(\pi)_{i, j}^{p r(n)}$ & $\begin{array}{l}\text { the production time of the } n \text {-th product in the machine placed in the } i \text {-th row of the } j \text {-th column in the } \\
\pi \text {-th plant }\end{array}$ \\
\hline$T(\pi)_{p r}^{n}$ & the matrix of manufacturing times in the $\pi$-th manufacturing plant \\
\hline \begin{tabular}{r|}
$\tau(\pi)_{e_{i, j} \rightarrow b_{i, j}}^{n}$ \\
$b_{i, j} \rightarrow e_{i^{\prime}, j^{\prime}}$ \\
$e_{i, j,} \rightarrow e_{i^{\prime}, j^{\prime}}$ \\
\end{tabular} & $\begin{array}{c}\text { the time of transporting the } n \text {-th product to its buffer store }\left(e_{i, j} \rightarrow b_{i, j}\right) \text {, from the buffer store to the } \\
\text { subsequent machine }\left(b_{i, j} \rightarrow e_{i^{\prime}, j^{\prime}}\right) \text { or directly to the subsequent machine if there is no buffer store for } \\
\text { this operation }\left(e_{i, j} \rightarrow e_{i^{\prime}, j^{\prime}}\right)\end{array}$ \\
\hline$\tau(\pi)_{\begin{array}{c}W \rightarrow b_{i, 0} \\
b_{i, 0} \rightarrow e_{i, 1} \\
W \rightarrow e_{i, 1}\end{array}}$ & $\begin{array}{l}\text { the time of transporting the } n \text {-th product from the store of charges to the first buffer store }\left(W \rightarrow b_{i, 0}\right) \text {, } \\
\text { from the first buffer store to the first machine }\left(b_{i, 0} \rightarrow e_{i, 1}\right) \text { or directly to the first machine }\left(W \rightarrow e_{i, 1}\right)\end{array}$ \\
\hline $\begin{array}{c}\tau(\pi)_{e_{I, J} \rightarrow b_{I, J}}^{n} \\
b_{I, J} \rightarrow Z \\
e_{I, J} \rightarrow Z \\
\end{array}$ & $\begin{array}{l}\text { the time of transporting the } n \text {-th product to the last buffer store }\left(e_{I, J} \rightarrow b_{I, J}\right) \text {, from the buffer store to } \\
\text { the matrix of orders }\left(b_{I, J} \rightarrow Z\right) \text { or directly to the matrix of orders }\left(e_{I, J} \rightarrow Z\right)\end{array}$ \\
\hline$T(\pi)_{t r}^{n}$ & $\begin{array}{l}\text { the matrix of transporting times of the } n \text {-th element within the } \pi \text {-th manufacturing system during the } \\
\text { production process }\end{array}$ \\
\hline$\tau(\pi)_{i, j}^{r e p l}$ & the replacement time of the tool in the machine placed in the $i$-th row of the $j$-th column \\
\hline$T(\pi)_{\text {repl }}$ & the matrix of replacement times of tools times in the $\pi$-th manufacturing plant \\
\hline$Z^{k}$ & the matrix of orders at the $k$-th stage \\
\hline$z_{m, n}^{k}$ & the $n$-th order for the $m$-th customer at the $k$-th stage \\
\hline$Y$ & the capacity matrix of buffer stores \\
\hline$v\left(b_{i, j}^{\pi}\right)_{n}$ & $\begin{array}{l}\text { the capacity of the buffer store placed behind the } j \text {-th column in the } i \text {-th row in the } \pi \text {-th manufacturing } \\
\text { plant for the } n \text {-th product }\end{array}$ \\
\hline$\pi$ & the $\pi$-th manufacturing subsystem \\
\hline$\Pi$ & the number of available manufacturing plants \\
\hline$\Xi$ & the adjustment matrix \\
\hline$\xi(m, n) \rightarrow \pi$ & adjustment of the $n$-th order of the $m$-th customer to the $\pi$-th manufacturing plant \\
\hline$\Delta$ & the distance matrix \\
\hline$\delta_{m \rightarrow \pi}$ & the distance from the $m$-th customer to the $\pi$-th manufacturing plant \\
\hline$\varphi_{\text {dif }}$ & the difference coefficient \\
\hline$\varphi_{f_{c}}$ & the fixed cost coefficient \\
\hline$\varphi_{h c}$ & the hidden cost coefficient \\
\hline$\varphi_{\text {per }}$ & the period coefficient \\
\hline
\end{tabular}




\section{Conclusion}

Each contemporary manufacturing task requires the so-called lean approach in order to minimise its fixed and operating costs, and so this paper focuses on the issue of modelling the complex logistics manufacturing system which consists of a defined number of subsystems. It is assumed that the subsystems are identical in nature and are equipped with identical machines which carry out the same operations. However, each subsystem is characterised by different total costs. This lets the operator to direct customers' orders to the subsystem whose total costs are the lowest in case of making the given order matrix, on the condition that the required subsystem is available. The main goal of the paper was to analyse the problem of modelling the complex logistics manufacturing system which is to meet the needs of the contemporary lean approach to production matters. The mathematical model (including necessary assumptions of the logistics-business system) was derived from the real logistics manufacturing system. The manufacturing strategies as well as heuristic algorithms responsible for controlling the course of the manufacturing process were proposed. The logistics manufactuing system was modelled by the iteration software engineering method which led to the required simulator of the logistics manufacturing system being created. The case study illustrates the method of choosing the most adequate manufacturing subsystem for making the order on the basis of cost calculation. The analysis was carried out for three sample manufacturing plants. The cost analysis decided which subsystem to choose, on the condition that the criterion of minimising total manufacturing costs is met. Moreover, it shows the influence of decreasing fixed costs on the total costs of making customers' orders.

Nevertheless, the total costs can be lowered e.g. by replacing human labour in the manufacturing industry. One of the possibilities is employing cobots which are robots intended to physically interact with humans in a shared workspace. However, this solution requires investments which are paid off in a defined period of time. Another option is searching for reducing fixed and operating costs by means of lean methods. However, it is worth considering whether or not to make costly investments in case of shortening the production series of customers' orders.

\section{Acknowledgement}

The authors are also grateful to the project No. CZ.02.2.69/0.0/0.0/16_027/0008521 "Support of International Mobility of Researchers at SU" which supports international cooperation.

\section{References}

[1] M. P. Fanti et al., "A Simulation Based Decision Support System for Logistics Management", Journal of Computational Science, vol. 10, pp. 86-96, 2015.

http://dx.doi.org/10.1016/j.jocs.2014.10.003

[2] H. S. Piao and X. Yao, "Simulation and Optimisation of the Cross-Docking Operation Scheme of Logistics Center", in Conference Proceedings of 2017 3rd IEEE International Conference on Control Science and Systems Engineering (ICCSSE), 2017, pp. 774-779.

[3] M. Siebert et al., "Lot Targeting and Lot Dispatching Decision Policies for Semiconductor Manufacturing: Optimisation under Uncertainty with Simulation Validation", Int. J. of Production Research, vol. 56, no. 1-2, pp. 629-641, 2018. http://dx.doi.org/10.1080/00207543.2017.1387679

[4] E. Simon et al., "Adapting Petri Nets to Des: Stochastic Modelling of Manufacturing Systems", International Journal of Simulation Modelling, vol. 17, no. 1, pp. 5-17, 2018.

http://dx.doi.org/10.2507/IJSIMM17(1)403

[5] M. R. Tu et al., "IoT-Based Production Logistics and Supply Chain System - Part 1 Modeling IoTbased manufacturing IoT supply chain", Industrial Management \& Data Systems, vol. 118, no. 1, pp. 65-95. http://dx.doi.org/10.1108/IMDS-11-2016-0503

[6] A. Yadav and S. C. Jayswal, "Modelling of Flexible Manufacturing System: A Review", Int. J. of Production Research, vol. 56, no. 7, pp. 2464-2487, 2018.

http://dx.doi.org/10.1080/00207543.2017.1387302

[7] J. Barbosa and P. Leitao, "Simulation of Multiagent Manufacturing Systems Using Agent-based Modelling Platforms", in Proc. of 9th IEEE International Conference on Industrial Informatics (INDIN), 2011.

[8] R. Sperka, "Application of a Business Economics Decision-Making Function in an Agent Simulation Framework", in Proc. Agent and Multi-Agent Systems: Technology and Applications, Kes-Amsta 2016, 2016, pp. 209-218. http://dx.doi.org/10.1007/978-3-319-39883-9_17

[9] J. Y. Zhao et al., "Simulation of Steel Production Logistics System Based on Multi-Agents", Int. 
J. of Simulation Modelling, vol. 16, no. 1, pp. 167-175, 2017.

http://dx.doi.org/10.2507/IJSIMM16(1)CO4

[10] G. G. Lim et al., "Multi-Agent Based Simulation for Evaluation of Mobile Business Models", Information - An International Interdisciplinary Journal, vol. 14, no. 9, pp. 3063-3080, 2011.

[11] K. Nagadi et al., "A Hybrid Simulation-Based Assessment Framework of Smart Manufacturing Systems", Int. J. of Computer Integrated Manufacturing, vol. 31, no. 2, pp. 115-128, 2018. http://dx.doi.org/10.1080/0951192X.2017.1407449

[12] M. Imran et al., "Cell Formation in a Cellular Manufacturing System Using Simulation Integrated Hybrid Genetic Algorithm", Computers \& Industrial Engineering, vol. 1058, pp. 123-135, 2017.

http://dx.doi.org/10.1016/j.cie.2016.12.028

[13] P. Kvasnica and I. Kvasnica, "Distributed Mathematical Model Simulation on a Parallel Architecture", Journal of Computing and Information Technology, vol. 20, no. 2, pp. 61-68, 2012. http://dx.doi.org/10.2498/cit.1001771

[14] B. Chramcov, "The Optimisation of Production System Using Simulation Optimisation Tools in Witness", Int. J. of Mathematics and Computers in Simulation, vol. 7. no. 2, pp. 95-105, 2013.

[15] A. Fazlirad and T. Freiheit, "Application of Model Predictive Control to Control Transient Behavior in Stochastic Manufacturing System Models", Journal of Manufacturing Science and Engineering-Transactions of the Asme, vol. 138, no. 8, 2016. http://dx.doi.org/10.1115/1.4031497

[16] A. Ferjani et al., "A Simulation-Optimisation Based Heuristic for the Online Assignment of Multi-Skilled Workers Subjected to Fatigue in Manufacturing Systems", Computers \& Industrial Engineering, vol. 112, pp. 663-674, 2017. http://dx.doi.org/10.1016/j.cie.2017.02.008

[17] J. E. Diaz et al., "Integrating Meta-Heuristics, Simulation and Exact Techniques for Production planning of a Failure-Prone Manufacturing System", European Journal of Operational Research, vol. 266, no. 3, pp. 976-989, 2018. http://dx.doi.org/10.1016/j.ejor.2017.10.062

[18] J. L. He et al., "Simulation-Based Heuristic Method for Container Supply Chain Network Optimisation", Advanced Engineering Informatics, vol. 29, no. 3, pp. 339-354, 2015.

http://dx.doi.org/10.1016/j.aei.2014.08.001

[19] A. Azadeh et al., "Design of Integrated Information System and Supply Chain for Selection of New Facility and Suppliers by a Unique Hybrid Meta-Heuristic Computer Simulation Algorithm", Int. J. of Advanced Manufacturing Technology, vol. 71, no. 5-8, pp. 775-793, 2014. http://dx.doi.org/10.1007/s00170-013-5417-8
[20] S. Bergmann et al., "On the Use of Artificial Neural Networks in Simulation-Based Manufacturing Control", Journal of Simulation, vol. 8, no. 1, pp. 76-90, 2014. http://dx.doi.org/10.1057/jos.2013.6

[21] G. C. Vosniakos et al., "Neural Network Simulation Metamodels and Genetic Algorithms in Analysis and Design of Manufacturing Cells", Int. J. of Advanced Manufacturing Technology, vol. 29, no. 5, pp. 541-550, 2006.

http://dx.doi.org/10.1007/s00170-005-2535-y

[22] T. Chen and Y. C. Wang, "Estimating Simulation Workload in Cloud Manufacturing Using a Classifying Artificial Neural Network Ensemble Approach", Robotics and Computer-Integrated Manufacturing, vol. 38, pp. 42-51, 2016. http://dx.doi.org/10.1016/j.rcim.2015.09.011

[23] T. Aljuneidi and A. A. Bulgak, "A Mathematical Model for Designing Reconfigurable Cellular Hybrid Manufacturing-Remanufacturing Systems", Int. J. of Advanced Manufacturing Technology, vol. 87, no. 5-8, pp. 1585-1596, 2016. http://dx.doi.org/10.1007/s00170-016-9141-z

[24] E. Asgari et al., "Proposing an Assignment Mathematical Model in Assembly Line Manufacturing System with Considering Human Factors' Role in Product Quality", in Proc. of IEEE International Conference on Industrial Engineering and Engineering Management (IEEM), 2017, pp. 1551-1555.

[25] A. G. Uriarte et al., "Improving the Material Flow of a Manufacturing Company via Lean, Simulation and Optimisation", in Proc. of IEEE International Conference on Industrial Engineering and Engineering Management (IEEM), 2017, pp. 1245-1250.

[26] O. Omogbai and K. Salonitis, "Manufacturing System Lean Improvement Design Using Discrete Event Simulation", Factories of the Future in the Digital Environment, vol. 57, pp. 195-200, 2016. http://dx.doi.org/10.1016/j.procir.2016.11.034

[27] M. Aghajani et al., "A Mathematical Programming Model for Cellular Manufacturing System Controlled by Kanban with Rework Consideration", Int. J. of Advanced Manufacturing Technology, vol. 83, no. 5-8, pp. 1377-1394, 2016. http://dx.doi.org/10.1007/s00170-015-7635-8

[28] R. De Smet and L. Gelders, "Using Simulation to Evaluate the Introduction of a Kanban Subsystem within an MRP-Controlled Manufacturing Environment", Int. J. of Production Economics, vol. 56-7, pp. 111-122, 1998. http://dx.doi.org/10.1016/S0925-5273(97)00095-9

[29] H. Aytug and C. A. Dogan, "A Framework and a Simulation Generator for Kanban-Controlled Manufacturing Systems", Computers \& Industrial Engineering, vol. 34, no. 2, pp. 337-350, 1998. http://dx.doi.org/10.1016/S0360-8352(97)00280-5 
[30] F. Longo and S. Lazzolino, "Agile Software Development: A Modeling and Simulation Showcase in Military Logistics", in Proc. of 4th International Conference in Software Engineering for Defence Applications, SEDA 2015, 2015, pp. 133-144.

http://dx.doi.org/10.1007/978-3-319-27896-4_12

[31] P. Suchanek and R. Bucki, "Business Process Modeling of Logistics Production Systems", in Proc. of 10th KES Conference on Agent and Multi-Agent Systems - Technologies and Applications (KES-AMSTA), 2016, pp. 199-207. https://link.springer.com/chapter/10.1007/978-3 -319-39883-9_16
Received: July 2018

Revised: February 2019

Accepted: March 2019

Contact addresses:

Robert Bucki

School of Business Administration in Karvina Silesian University in Opava Czech Republic e-mail: bucki@opf.slu.cz

Petr Suchánek School of Business Administration in Karvina Silesian University in Opava Czech Republic e-mail: suchanek@opf.slu.cz

ROBERT BUCKI is an experienced researcher whose main interests are designing information logistics systems; modeling economic systems; simulation of economic systems; optimization of economic, logistics and production systems; software engineering; logistics systems in companies; operational research, modeling and simulation; implementation of information systems; information tools in logistics processes and international logistics systems. He is the author and co-author of more than 80 scientific works including monographs, chapters in monographs, conference papers and articles in scientific journals which are indexed in Scopus and Web of Science.

PETR SuCHÁNEK is an experienced researcher dealing with implementation and integration of IS/IT into business environments and the public sector. Specifically, he has been involved in solving issues in modelling, mathematical modelling and simulation of socio-economic systems such as e-business systems, logistic systems, manufacturing systems, as well as measuring the benefits of IS/IT, especially in business. $\mathrm{He}$ is author or co-author of more than 80 scientific works including monographs, book chapters, as well as journal and conference papers indexed both in Scopus and Web of Science bases. 